\title{
http://bjas.journals.ekb.eg Role of Transcranial Doppler Assessment of Cerebral Blood Flow in Neonatal Sepsis
}

M.M.Refaat, A.A.Torky and D.A.Gouda

Radiology Dept., Faculty of Medicine, Benha Univ,Benha, Egypt

E-Mail:doaa.goudasalem@gmail.com

\begin{abstract}
Neonatal sepsis is one of the leading causes of morbidity and mortality among term and preterm infants worldwide.assessment of the changes in cerebral blood flow velocity [CBFV] in cases of the neonatal sepsis. This study including 100 infants. 60neonates are diagnosed with neonatal sepsis [Sepsis group]. while the other 40 neonates with no signs of neonatal sepsis [Control group]. Trans-cranial Doppler [TCD] was carried out for assessment of blood flow velocity in the anterior [ACA] and middle cerebral arteries [MCA] Peak systolic velocity [PSV], end diastolic velocity [EDV], and resistive index [RI] were measured.RI and PI were significantly decreased, with mean RI 0.56in MCA \& Median RI in ACA 0.59 \& Median PI 0.98; 0.98 in MCA \& ACA respectively in the case group, while with a mean RI 0.75 in MCA \& Median RI 0.72 in ACA respectively \& Median PI1.35; 1.27 in MCA \& ACA respectively . Whereas the Peak systolic velocity was increased with a mean of 69.07 in MCA \& Median of 66.4 in ACA respectively in case group \& mean of 50.22 ; in MCA \& Median of 44.0 in ACA in control group with p value $<0.001$ in MCA \& ACA respectively. neonatal sepsis causes increase in Peak systolic velocity \& decrease in the Resistive \& Pulsatility index thus causing increase in the cerebral blood flow, which in turn showed increased incidence in Intraventricular hemorrhage .
\end{abstract}

Keywords Neonatal sepsis, Cerebral blood flow [CBF], Trans-cranial Doppler [TCD].

\section{Introduction}

The advent of cranial ultrasound in neonatology has greatly improved our knowledge of the presence and incidence of brain lesions in newborn infants. It is used routinely for infants at risk of neurological impairment, such as those born prematurely or who have suffered from birth asphyxia [1].

Cerebral blood flow, as measured by Doppler ultrasound, has been studied mainly to help determine whether alterations have occurred in the neonatal cerebral circulation that could result in brain damage and adverse developmental outcomes. Establishment of the predictive validity of cerebral blood flow measures by Doppler ultrasound is essential to ensure their usefulness in the early assessment and interpretation of hemodynamic changes [2].

Neonatal sepsis is one of the leading causes of morbidity and mortality among term and preterm infants worldwide [3].

Although advances in neonatal care have improved survival and reduced complications, survivors of neonatal sepsis are still vulnerable to short and long-term neurodevelopment morbidity [4].

Hospital-born babies in developing countries are at increased risk of neonatal infections because of poor intrapartum and postnatal infection-control practices [5]

Neonatal sepsis is defined classically as a clinical syndrome characterized by systemic signs of infection frequently accompanied by bacteremia [6] .

Two types of neonatal sepsis have been observed: early-onset disease, when features of sepsis appear during the first $72 \mathrm{~h}$ of birth; and late-onset disease, where the disease manifests beyond $72 \mathrm{~h}$. Whereas the latter is considered to be acquired after birth, early-onset neonatal sepsis [EONS] is acquired in utero resulting in chorioamnionitis [7].

There is paucity of literature regarding the early alteration of cerebral blood flow $[\mathrm{CBF}]$ in neonatal sepsis. The brain of the newborn is highly susceptible to blood flow fluctuations. Moderately elevated $\mathrm{CBF}$ can in-crease the risk of cerebral hemorrhage whereas moderate hypoperfusion can expose the brain to ischemic damage [8].

\section{Patients and Methods}

This is a prospective cohort study,was conducted in the Neonatal Intensive Care Unit [NICU] of Benha Children Hospital during the period from October 2018 to April 2019. Approval of research ethics committee was obtained before conducting the study. Informed written consent was obtained from the parents.

\subsection{Population of study}

The study was carried out on two groups: Group [I] Sixty neonates were clinically \& laboratory diagnosed with neonatal sepsis [Sepsis group]. Group [II] Forty neonates with no signs of early onset neonatal sepsis [Control group].All of them were preterm with range from 29-35 weeks, birth weight $<2000 \mathrm{gm}, \&$ normal labs other for those of sepsis in the sepsis group.For the sepsis group we enrolled those who met the inclusion \& exclusion criteria.

\subsection{Inclusion criteria}


We included neonates with: Laboratory criteria [WBC $<5000$ or $>20,000 \times 109$ cells/L; I/T ratio > 0.2 ; platelet count $<100,000 \times 109 / \mathrm{L}$. quantitative C-reactive protein [CRP] levels $\geq 6$ $\mathrm{mg} / \mathrm{L}$, with or without positive blood culture results. And who showed clinical symptoms \& signs of the following: Respiratory compromise: tachypnea, increased apnea and increased desaturations .Cardiovascular compromise: bradycardia, pallor, hypotension.Metabolic changes: hypothermia, hyperthermia, feeding intolerance, glucose instability, metabolic acidosis.Neurological changes: lethargy, hypotonia, decreased activity.

\subsection{Exclusion criteria}

Perinatal asphyxia, congenital infections, Respiratory distress syndrome, Meconium aspiration syndrome, congenital heart disease, Hemolytic anemia, metabolic disorders, surgical disorders, Gross congenital malformations.

Neonates with altered arterial blood gas [ABG] parameters, hemodynamic instability, mechanical ventilation, at the time of CBF assessment.

\subsection{Clinical work-up}

Perinatal history, clinical examination which was made at birth to exclude any systemic disease or congenital malformation, laboratory estimation of blood glucose, serum electrolytes, coagulation profile, liver and kidney function tests, ABG, tests to exclude inborn errors of metabolism were recorded. Blood culture was taken, Transcranial ultrasound [TCU] was performed \& cerebral blood flow $[\mathrm{CBF}]$ was assessed by Transcranial Doppler [TCD] ultrasound.

\subsection{Assessment of cerebral blood flow}

Table(1) Comparison between case and control groups according gender, age , CRP, weight WBCs, Platelets, MCA [PSV,RI,PI ],ACA [PSV,RI,PI] .

\begin{tabular}{lcccccc}
\hline & \multicolumn{2}{c}{ Case group [60] } & \multicolumn{2}{c}{ Control group [40] } & $\begin{array}{c}\text { Statistical } \\
\text { test }\end{array}$ & P value \\
\cline { 2 - 5 } Gender & No & $\mathbf{\%}$ & No & $\mathbf{\%}$ & & \\
Male & & & & & & \\
Female & 40 & 66.7 & 20 & 50.0 & $\mathrm{X}^{2}=2.78$ & 0.096 \\
& 20 & 33.3 & 20 & 50.0 & & \\
Age /year & Median & IQR & Median & IQR & MW test & \\
CRP & 32.5 & $32.0-34.0$ & 32.0 & $32.0-34.0$ & 0.87 & 0.38 \\
& 66.0 & $50.0-82.0$ & 3.5 & $2.0-4.0$ & 8.47 & $<0.001^{* *}$ \\
Weight [kg] & Mean & SD & Mean & SD & St t test & \\
WBCs & 1.63 & 0.14 & 1.64 & 0.13 & 0.57 & 0.57 \\
Platelets & 24.94 & 7.37 & 14.87 & 2.07 & 8.42 & $<0.001^{* *}$ \\
MCA PSV & 320.83 & 113.63 & 274.4 & 103.09 & 2.08 & $0.04^{*}$ \\
MCA RI & 69.07 & 12.64 & 50.22 & 11.81 & 7.5 & $<0.001^{* *}$ \\
& 0.56 & 0.08 & 0.75 & 0.05 & 12.75 & $<0.001^{* *}$ \\
MCA PI & Median & IQR & Median & IQR & MW test & \\
ACA PSV & 0.98 & $0.82-1.06$ & 1.35 & $1.26-1.48$ & 7.61 & $<0.001^{* * *}$ \\
ACA RI & 66.4 & $61.3-69.9$ & 44.0 & $35.3-50.7$ & 5.97 & $<0.001^{* *}$ \\
\hline
\end{tabular}


\begin{tabular}{lrr}
\hline ACA PI & 0.98 & $0.68-1.02$ \\
\hline and PI were significantly decreased, with
\end{tabular} mean RI 0.56in MCA \& Median RI in ACA0.59 respectively \& $p$ value $<0.001 \&$ Median PI 0.98 ; 0.98 in MCA \& ACA respectively in the case group, while with a mean RI0.75 in MCA \& Median RI 0.72 ACA respectively \& Median PI1.35; 1.27 in MCA \& ACA respectively \& a p

$1.27 \quad 1.25-1.39 \quad 6.74 \quad<0.001^{* *}$

value $<0.001$. Whereas the Peak systolic velocity was increased with a mean of 69.07 in MCA \& Medianof 66.4 in ACA respectively in case group $\&$ mean of 50.22 ; in MCA \& Medianof 44.0 in ACA in control group with $\mathrm{p}$ value $<0.001$ in MCA \& ACA respectively.

Table(2) shows distribution of case group according to IVH and death.

\begin{tabular}{lll}
\hline [60] & NO & \% \\
\hline IVH & & \\
GI & 8 & 13.3 \\
GIII & 2 & 3.3 \\
GIV & 2 & 3.3 \\
Death & & \\
Yes & 6 & 10.0 \\
No & 54 & 90.0 \\
\hline
\end{tabular}

Among the sepsis group, 12 patients [19.9\%] showed signs of IVH/ GMH in cranial ultrasonography during their hospital stay \& 6 of them [10\%] [6\% of total no. of cases] died during their hospital stay.

\section{Case Presentation}

Gestational age 30 weeks ,Birth weight 1700 gm. , Clinical \& proven sepsis by blood culture.

\section{MCA}

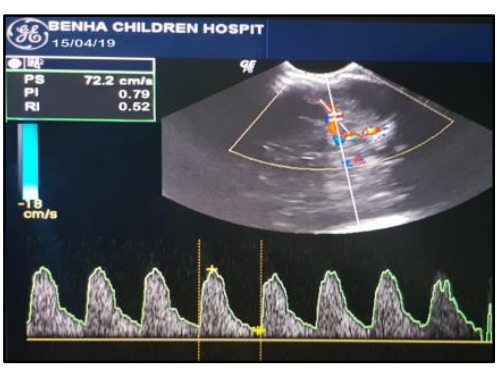

Fig (1) Doppler image of MCA case 1.

\section{Showing}

- Peak systolic velocity [PSV] 72.2

- Resistive Index [RI] 0.52

- Pulsatile Index [PI] 0.79

ACA

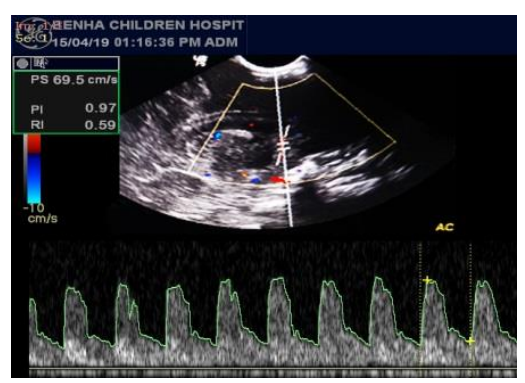

Fig (2) Doppler image of ACA case 1.

\section{Showing}

- Peak systolic velocity [PSV] 69.5

- Resistive Index [RI] 0.59

- Pulsatile Index [PI] 0.97 Opinion

- Increase in PSV.

- Decrease in Decrease in RI \& PI.

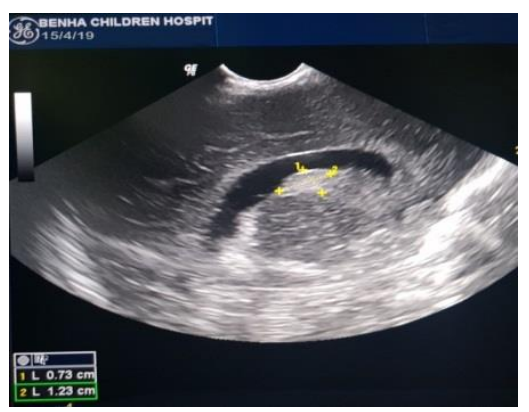

Fig (3) Sagittal image shows IVH GI in case 1.

Five Days Later Complicated By Intra Ventricular Hemorrhage grade [I].

\section{Discussion}

Worldwide annual 1 million neonatal deaths is attributed to neonatal sepsis alone, $99 \%$ of the world's neonatal deaths occur in low-income and middle-income countries [9].

Neonatal sepsis is a clinical syndrome characterized by signs and symptoms of infection with or without accompanying bacteremia in the first month of life. It encompasses various systemic infections of the newborn such as septicemia, meningitis, pneumonia, arthritis, osteomyelitis, and urinary tract infections [10].

The stress, and cytokines on glial cells have been speculated to be the contributory exact nature of cellular and molecular mechanisms of sepsis- 
induced encephalopathy is still elusive. The impairment of cerebral autoregulation, damage to blood-brain barrier [BBB], and the direct inflammatory effects of free radicals, oxidative factors [11].

The exact mechanism by which these cytokines access to the cerebral compartment to induce injury is still debatable. Transfer of cytokines across BBB, and damage of BBB causing increased permeability [12] .could be the responsible factors. It has also been shown that astrocytes and microglia are capable of producing proinflammatory cytokines during inflammation [13].

There is paucity of literature regarding the early alteration of cerebral blood flow $[\mathrm{CBF}]$ in neonatal sepsis. The brain of the newborn is highly susceptible to blood flow fluctuations. Moderately elevated $\mathrm{CBF}$ can increase the risk of cerebral hemorrhage whereas moderate hypo perfusion can expose the brain to ischemic damage [14].

Changes in cerebral blood flow velocity [CBFV] may have a key role in perinatal brain damage, and both acute and long-term morbidity may be closely related to rapid vascular changes during the early hours of life. Moreover, altered CBF may also be used as a surrogate marker for the diagnosis of EONS also other literature investigated the association of potential risk factors for neurodevelopmental disorders. Children who developed neonatal sepsis were three times more likely to have neuromotor and cognitive development alterations at 12 months of corrected age [15].

More over Pathogenesis of IVH is multifactorial and is primarily ascribed to a] inherent fragility of the germinal matrix vasculature, b] disturbance in the cerebral blood flow [16].

Cranial sonography plays an important role in the initial evaluation of infants with suspected bacterial meningitis and in monitoring for complications of the disease [17] \& Color and duplex Doppler sonography have broadened applications in pediatric neurosonography for evaluation of cerebral vascular anatomy and perfusion, It has proven diagnostic value in detecting the most common brain lesions in premature neonates; such lesions include those due to intraventricular hemorrhage and white matter disease, the technique is readily applied at bedside, does not involve ionizing radiation, is cost effective and can be used sequentially[18].

Improvements in critical care medicine have resulted in the increased survival of premature, low birth weight and asphyxiated infants. An integrated clinical and radiological approach to the neurological assessment of such neonates over the past few years has led to a better understanding of the cause and evolution of the cerebral lesions and thus allowed more appropriate medical intervention to favourably alter clinical outcome[18].

Higher peak systolic velocity [PSV] in all the three major cerebral vessels Significantly lower resistance [RI and PI], vasodilatation, and higher peak systolic velocity [PSV] in MCA \& ACA have been noted in neonates with EONS in comparison to neonates of the control group, indicating generalized increase in $\mathrm{CBF}$ as an early response to sepsis. This is agreed with the study of [19] , that detected significantly lower resistance [RI and $\mathrm{PI}$, vasodilatation, and [ICA, VA, and MCA] documented within $24 \mathrm{~h}$ of birth in neonates with EONS, indicating generalized increase in CBF as an early response to sepsis, $\boldsymbol{\&}$ [20] who stated that their chorioamnionitis [HC] infants had decreased resistance in most of the major cerebral vessels compared with controls, with $\mathrm{HC}$ males being more affected than $\mathrm{HC}$ females.

However in this study the Mean PSV was 69.07, which is higher than what was found by [19] which was 56.05, that could be related to the timing of the performance of the TCD, as they conducted it within the first 24 hours after delivery, while in this study was on the 3 rd day, \& it is documented that there is progressive increase in the CBFV with the post natal age [14].

We also found that there is a strong relation between neonatal sepsis, developing IVH \& subsequent death. As there were cases who developed IVH \& six of them died within the two months follow up period, also this was matched with the results of [19]who found and added that within the sepsis group, CBF was further increased in those who expired or had IVH/GMH compared to those who had uncomplicated survival, \& [20] who found that The ACA PSV in HC males was significantly negatively correlated with both motor and cognitive composite scores, as well as personal-social and problem-solving skills.

There is a paucity of literature demonstrating the effect of sepsis on CBF though early cerebrovascular alteration.[21], this increase in $\mathrm{CBF}$ may be a part of fetal response to chorioamnionitis [FIRS]. [22].

Whereas other authors have demonstrated decreased CBFV along with higher PI in patients with sepsis[23] and[24]. Reduced CBF is ascribed to the vasoconstriction of the resistance arterioles [23]. Increased PI, a parameter for compliance of the vascular bed suggesting cerebral edema or venous congestion, has been found to correlate with poor neurological outcome. [25]

We have used TCD ultrasound to measure $\mathrm{CBFV}$ as it allows repeated and safe non-painful bedside assessment of CBF.

The strength of this study was in the strict inclusion and exclusion criteria which was followed. Neonates with perinatal asphyxia, 
vasomotor instability, mechanical ventilation, altered ABG parameters like hypo-/ hyperoxia, hypo-/ hypercarbia and acidosis, and those receiving caffeine / theophylline/ anticonvulsants at the time of TCD examination were excluded, as these factors could have influence CBFV measurements, \& normothermia was maintained.

However, the present study was not without limitation. We attempted for a cross sectional single-time assessment of CBFV and, therefore, could not assess the fluctuation of CBFV over a period of time \& correlate it with the adverse effect.

To conclude, in the present study, vasodilatation and increased $\mathrm{CBF}$ in early hours of sepsis, have been found. In some literature it was proved to have immediate and late, long-term consequences \& Significant association was observed between sepsis and increased CBF, Among the sepsis group, mortality and intracranial complications were associated with higher CBF. Altered CBFV may be considered as an important additional investigation and utilized as therapeutic target for management in sepsis. Care should be taken to maintain normotension and avoid activities which can cause alteration of blood flow.

\section{Conclusion}

neonatal sepsis causes increase in Peak systolic velocity \& decrease in the Resistive \& Pulsatility index thus causing increase in the cerebral blood flow, which in turn showed increased incidence in Intraventricular hemorrhage among the sepsis group compared with the control group.

\section{References}

[1] Ng. VWK and JE. MacSweeny, Ultrasound of the neonatal brain $\mathrm{Br}$ Jhosp Med,Vol.57,PP.19-022,2001.

[2] Wu. Ying-Chin, Hsieh. Wu-Shiun, Hsu .Chyong-Hsin, Chiu .Nan-Chang, Chou. Hung-Chieh, Chen. Chien-Yi, Peng .ShinnForng, Hung. Han-Yang, Chang. Jui-Hsing, Wei J. Chen, Jeng .Suh-Fang, Relationship of Neonatal Cerebral Blood Flow Velocity Asymmetry with Early Motor, Cognitive and Language Development in Term Infants Ultrasound in Medicine \& Biology;Vol.39,PP.5-797-803 ,2013.

[3] A .Gullo, N. Bianco, , G. Berlot, Management of severe sepsis and septic shock: challenges and recommendations. Crit. Care Clin,Vol. 22,PP. 489-501,2006.

[4] N. Chaudhry, A. K. Duggal, Sepsis associated encephalopathy. Adv. Med,vol 23,PP. 762320,2014 .

[5] KM. Anita Zaidi, W. Charles Huskinsb, c. Durrane Thaver, A.MBBS, A.
Zulfiqar Bhutta, MD.Zohair Abbas, A. Donald Goldmann, Hospital-acquired neonatal infections in developing countries. The Lancet,Vol. 365,PP.1175-1188,2005.

[6] Maria. Regina Bentlin, Lígia .Maria Suppo de Souza Rugolo, Late-onset Sepsis: Epidemiology, Evaluation, and Outcome, Vol.10,PP.8,2010.

[7] P. Toti, C .De Felice, R. Occhini, K .Schuerfeld, M .Stumpo, MC. Epistolato, R .Vatti, G. Buonocore, Spleen depletion in neonatal sepsis and chorioamnionitis. Am J Clin Pathol,Vol. 122,PP.765-771,2004.

[8] M. Pezzati, C. Dani, R .Biadaioli, L .Filippi, R. Biagiotti, T. Giani, FF.Rubaltelli ,Early postnatal Doppler assessment of cerebral blood flow velocity in healthy preterm and term infants. Dev Med Child Neurol,Vol. 44,PP.745-752,2002.

[9] JE. Lawn, Katarzyna. WilczynskaKetende and N .Simon Cousens, Estimating the causes of 4 million neonatal deaths in the year 2000. Int. J. Epidemiol, Vol. (35),PP. 706$718,2006$.

[10] MJ .Sankar, R .Agarwal, AK. Deorari, VK .Paul, The Indian Journal of Pediatrics. Sepsis in newborn, Vol.70,PP. 261-266,2008.

[11] J. G.Shatrov, S. C.Birch, L. T.Lam, J. A.Quinlivan, S.McIntyre, G.L.Mendz, Chorioamnionitis and cerebral palsy: a metaanalysis. Obstetrics \& Gynecology,Vol.116(2),PP. 387-392,2010.

[12]R. W.Redline, O.Faye-Petersen, D.Heller, F.Qureshi, V.Savell, C.Vogler, Society for Pediatric Pathology, Perinatal Section, Amniotic Fluid Infection Nosology Committee. Amniotic infection syndrome: nosology and reproducibility of placental reaction patterns. Pediatric and Developmental Pathology,Vol. 6(5),PP.435-448,2003.

[13] M.Kehrer, R.Goelz, I.Krägeloh-Mann, M.Schöning, Measurement of volume of cerebral blood flow in healthy preterm and term neonates with ultrasound. The Lancet,Vol.360(9347),PP. 1749-1750,2002.

[14] M .Pezzati, C. Dani, R. Biadaioli, L. Filippi, R .Biagiotti, $\mathrm{T}$.Giani, FF .Rubaltelli, Early postnatal Doppler assessment of cerebral blood flow velocity in healthy preterm and term infants. Dev Med Child Neurol;Vol.44,PP.745-752,2002.

[15] U.Kiechl-Kohlendorfer, E.Ralser, U. P.Peglow, G.Reiter, R. Trawöger, Adverse neurodevelopmental outcome in preterm infants: risk factor profiles for different gestational ages. Acta paediatrica,Vol.98[5],PP. 792-796,2009.

[16]BALLABH, Praveen, Pathogenesis and prevention of intraventricular hemorrhage. Clinics in perinatology, Vol. 41.1,PP. 47- 
67,2014 .

[17] LL.Barr, Neonatal cranial ultrasound. Radiol Clin North AmJ;Vol.37(6),PP.11277911,2009.

[18] Ng. VWK and JE .MacSweeny, Ultrasound of the neonatal brain $\mathrm{Br}$ Jhosp Med;Vol.57,PP.19-22,2001.

[19]Basu .Sriparna , Shashikant Dewangan , Ram .Chandra Shukla , Shampa Anupurva , Ashok Kumar, Cerebral blood flow velocity in earlyonset neonatal sepsis and its clinical significance. Eur J Pediatr,Vol. 171,PP.901$909,2012$.

[20]FR .Koch, CL .Wagner, DD. Jenkins, MJ .Caplan, JK. Perkel, LG .Rollins, LD. Katikaneni and DM. Mulvihill, Sex differences in cerebral blood flow following chorioamnionitis in healthy term infants. Journal of Perinatology,Vol. 34,PP.197202,2014.

[21] Y.W. Wu, G. J.Escobar, J. K.Grether, L. A.Croen, J. D.Greene, T. B. Newman, Chorioamnionitis and cerebral palsy in term and near-term infants. Jama, Vol.290[20],PP. 2677-2684,2003.
[22]G .Rocha, E .Proença, C .Quintas, T .Rodrigues, $\mathrm{H}$.Guimaraes, Chorioamnionitis and brain damage in the preterm newborn. J Matern Fetal Neonatal Med,Vol.20,PP.745749,2007.

[23]BF .Matta, PJ. Stow, Sepsis-induced vasoparalysis does not involve the cerebral vasculature: indirect evidence from autoregulation and carbon dioxide reactivity studies. $\mathrm{Br} \quad \mathrm{J}$ Anaesth;Vol.76,PP.790794,1996.

[24] TD. Yanowitz, JA .Jordan, CH. Gilmour, R .Towbin, A .Bowen, JM .Roberts, BS .Brozanski,Hemodynamic disturbances in premature infants born after chorioamnionitis: association with cord blood cytokine concentrations. Pediatr Res,Vol.51,PP.310 316,2002 .

[25]DI. Altman, JM .Perlman, JJ. Volpe, WJ .Powers, Cerebral oxygen metabolism inewborns. Pediatrics, Vol.92,PP.99-104,1993. 